\title{
The Potential of Lymph Node Yield as a Quality Indicator of Esophagectomy for Esophageal Cancer
}

\author{
Satoru Matsuda, MD, PhD, and Yuko Kitagawa, MD, PhD, FACS \\ Department of Surgery, Keio University School of Medicine, Tokyo, Japan
}

Because of the abundant lymphatic routes and anatomic structures connecting the throat with the stomach, esophageal cancer is considered to be a dismal disease likely to involve extensive lymph node (LN) metastasis even in the early stage. ${ }^{1}$ To prevent the spread of cancer cells by the lymphatic route, surgical resection with extended LN dissection has been the standard procedure. ${ }^{2}$ Because the extent of LN metastasis may differ depending on the location of the primary tumor and tumor histology, identifying the appropriate field of LN dissection is critical to patient cure.

One of the quality indicators suggesting successful esophagectomy with relevant LN dissection is the number of LNs retrieved. ${ }^{3,4}$ To evaluate the utility of this indicator, a study assessed the prognostic impact from the number of LNs harvested. The findings showed a positive correlation between the number of LNs harvested and the outcome of the esophagectomy. ${ }^{5}$ Moreover, the number of LNs retrieved is associated with several patient and disease characteristics, including preoperative weight loss, low Charlson comorbidity score, and higher clinical N stage. In addition, stage migration, in which a lower number of LNs examined underestimates the pathologic stage, could exist. Therefore, a larger number of LNs dissected might not simply reflect the extent of lymphadenectomy. For an accurate evaluation of the correlation between LN yield and prognosis, the background data for each patient should be precisely matched to minimize confounding.

(C) Society of Surgical Oncology 2020

First Received: 25 August 2020

Accepted: 3 September 2020;

Published Online: 15 October 2020

Y. Kitagawa, MD, PhD, FACS

e-mail: kitagawa@a3.keio.jp
In the current issue of Annals of Surgical Oncology, we read with great interest how van der Werf et al. ${ }^{6}$ successfully showed that the number of LNs harvested was associated with more accurate staging but did not have an impact on patient survival. The authors reviewed national data using the Dutch Upper Gastrointestinal Cancer Audit combined with the National Healthcare Insurance Database and retrieved data for esophageal cancer patients who underwent esophagectomy after neoadjuvant chemoradiotherapy (NACRT). In their comprehensive analysis with propensity-score matching, esophageal cancer patients with more than 15 retrieved LNs exhibited a higher proportion of pathology-confirmed positive LN metastasis but showed no difference in survival, indicating that the pathologic stage was appropriately assigned in the high LN-yield group.

As noted by the authors, their result was inconsistent with previous findings of the relation between an increased number of LNs retrieved and a better survival outcome. They speculated that this discrepancy might reflect the fact that the current study was carried out after Dutch institutions had started various improvement processes. It appeared reasonable to believe that the reported LN yield might not be an effect of more extended LN dissection because general surgical procedures among the participating institutions had been standardized already.

The study focused on patients who received NACRT for advanced esophageal cancer, whose response to preoperative treatment would be a stronger prognostic factor for survival than the extent of local LN eradication. Meanwhile, as we reported previously, extensive LN dissection combined with thoracic duct resection improved survival, especially for patients with early esophageal cancer, for which perioperative treatment is not applied as standard of care. ${ }^{7}$ In our report, we compared the number of LNs dissected in transthoracic minimally invasive 
esophagectomy (TMIE) with or without thoracic duct resection and the number of LNs obtained by extended lymphadenectomy. As a result, we showed that extensive TMIE increased the LN yield and improved survival.

When the extent of lymphadenectomy between different surgical approaches is quantified, the number of LN retrieved can be a reliable surrogate marker. Therefore, the value of LN yields could differ depending on tumor biology, tumor advancement, and perioperative multidisciplinary treatment.

To date, surgical procedures for esophageal cancer have varied widely across countries with regard to extent of lymphadenectomy, implementation of minimally invasive approaches, and perioperative treatment. With the updated clinical evidence on the use of LN yield as a quality indicator of lymphadenectomy extension and pathologic diagnosis, we expect that the standardized surgical procedure that fits the patient's background and tumor characteristics will be established as an individualized multidisciplinary treatment for esophageal cancer patients.

DISCLOSURE Yuko Kitagawa received lecture fees from Asahi Kasei Co, Ltd; Taiho Pharmaceutical Company, Ltd; Chugai Pharmaceutical Company, Ltd; EA Pharma Company, Ltd; Yakult Honsha Company, Ltd; Otsuka Pharmaceutical Company, Ltd; Otsuka Pharmaceutical Factory Inc, Shionogi \& Company, Ltd; Astellas Pharma, Inc; Dainippon Sumitomo Pharma Company, Ltd; Taisho Toyama Pharmaceutical Company; Ono Pharmaceutical Company; Nihon Pharmaceutical Co, Ltd; Sanofi K.K., Eisai Company, Ltd; and Kaken Pharmaceutical Company, Ltd. Yuko Kitagawa received Grants for research expenses and scholarship from Taiho Pharmaceutical Company, Ltd; Chugai Pharmaceutical Company, Ltd; Yakult Honsha Company, Ltd; Daiichi Sankyo Company, Ltd; Merck Serono Company, Ltd; Asahi Kasei Company, Ltd; EA Pharma Company, Ltd; Otsuka Pharmaceutical Company, Ltd; Takeda Pharmaceutical Company, Ltd; Otsuka Pharmaceutical Factory, Inc; Shionogi Company, Ltd; Kaken Pharmaceutical Company, Ltd; Kowa Pharmaceutical Company, Ltd; Astellas Pharma, Inc; Medicon, Inc; Dainippon Sumitomo Pharma Company, Ltd; Taisho Toyama
Pharmaceutical Company, Ltd; Kyouwa Hakkou Kirin Company, Ltd; Pfizer Japan, Inc; Ono Pharmaceutical Company, Ltd; Nihon Pharmaceutical Company, Ltd; Japan Blood Products Organization Medtronic Japan Company, Ltd; Sanofi K.K., Eisai Company, Ltd; Tsumura \& Company, Ltd; KCI Licensing, Inc; Abbott Japan Company, Ltd; and Fujifilm, Toyama Chemical Company, Ltd. Yuko Kitagawa worked as a director of endowed chair supported from Taiho Pharmaceutical Company, Ltd; and Chugai Pharmaceutical Company, Ltd. Satoru Matsuda has no conflicts of interest.

\section{REFERENCES}

1. Takeuchi H, Fujii H, Ando N, et al. Validation study of radioguided sentinel lymph node navigation in esophageal cancer. Ann Surg. 2009;249:757-63.

2. Matsuda S, Takeuchi H, Kawakubo H, Kitagawa Y. Three-field lymph node dissection in esophageal cancer surgery. J Thorac Dis. 2017;9:S731-40.

3. van der Werf LR, Dikken JL, van Berge Henegouwen MI, et al. A population-based study on lymph node retrieval in patients with esophageal cancer: results from the Dutch upper gastrointestinal cancer audit. Ann Surg Oncol. 2018;25:1211-20.

4. Busweiler LA, Wijnhoven BP, van Berge Henegouwen MI, et al. Early outcomes from the Dutch Upper Gastrointestinal Cancer Audit. Br J Surg. 2016;103:1855-63.

5. Visser E, Markar SR, Ruurda JP, et al. Prognostic value of lymph node yield on overall survival in esophageal cancer patients: a systematic review and meta-analysis. Ann Surg. 2019;269:261-8.

6. van der Werf LR, Gisbertz SS, Wijnhoven BP, van Berge Henegouwen M. A propensity score-matched cohort study to evaluate the association of lymph node retrieval with long-term overall survival in patients with esophageal cancer. Ann Surg Oncol. 2020. https://doi.org/10.1245/s10434-020-09142-w. (in press).

7. Matsuda S, Kawakubo $\mathrm{H}$, Takeuchi $\mathrm{H}$ et al. Minimally invasive oesophagectomy with extended lymph node dissection and thoracic duct resection for early-stage oesophageal squamous cell carcinoma. Br J Surg. 2020;107:705-11.

Publisher's Note Springer Nature remains neutral with regard to jurisdictional claims in published maps and institutional affiliations. 